\title{
Ising model on a Cayley tree with competing and aperiodic interactions
}

\author{
R. F. S. Andrade \\ Instituto de Fisica, Universidade Federal da Bahia, 40210-340 Salvador, Brazil \\ S. R. Salinas \\ Instituto de Fisica, Universidade de São Paulo, Caixa Postal 66318, 05315-970 São Paulo, Brazil
}

(Received 25 February 1997)

\begin{abstract}
We include extra aperiodic interactions in the Ising model with competing ferromagnetic and antiferromagnetic interactions between first and second neighbors along the branches of a Cayley tree. The problem is formulated as a nonlinear map whose attractors correspond to solutions deep in the interior of the tree. We present some analytical and numerical calculations for aperiodic interactions introduced according to the rules of a Koch curve and a Fibonacci sequence. For small values of a parameter of aperiodicity, there are no relevant changes in the phase diagram, except for the appearance of some bumps along the paramagneticmodulated transition. As the aperiodic interactions become more important, there are several new phenomena, such as phase locking and phase splitting, and the enhancement of the regions of chaotic attractors and of co-stability of different structures. [S1063-651X(97)14307-0]

PACS number(s): $05.50 .+\mathrm{q}$
\end{abstract}

\section{INTRODUCTION}

The presence of competing interactions in magnetic and ferroelectric systems leads to complex phase diagrams with a variety of transition lines and modulated phases. The ANNNI model, which consists of an Ising spin Hamiltonian on a cubic lattice, with ferromagnetic interactions on the planes, and competing ferromagnetic and antiferromagnetic interactions between nearest and next-nearest neighbors along an axial direction, is known to reproduce some features of these complex phase diagrams [1]. Qualitatively similar phase diagrams can also be obtained from some counterparts of the ANNNI model on a Cayley tree [2]. These simple analogs of the ANNNI model can be formulated as a nonlinear dissipative map whose attractors correspond to physical solutions deep in the interior of the tree [3].

It is certainly interesting to investigate how the sequences of modulated phases and transition lines in these rich phase diagrams can be affected by the presence of random impurities (as random or diluted bonds or sites, for example). In the easier, and less relevant, case of annealed impurities, some mean-field calculations for the ANNNI model indicate slight changes in the transition lines, and the persistence of the overall structure of the modulated phases [4]. In the physically more relevant case of quenched impurities, there are some arguments to claim that the long-period commensurate phases are unstable in three dimensions [5]. Very recent calculations for the ANNNI model in a random field indicate almost no changes in weak fields, but a variety of new features as the field increases [6]. In the present paper, instead of looking at a really disordered situation, we mimic the effects of quenched impurities by considering an Ising model with deterministic but aperiodic competing interactions.

We use the Koch curve and the standard Fibonacci sequence to add extra deterministic but aperiodic interactions to the analog of the ANNNI model on a Cayley tree introduced by Yokoi, de Oliveira, and Salinas [3]. In Sec. II, we define the model, and formulate the problem according to a scheme of successive periodic approximations. In Sec. III, we obtain the borders of stability of the paramagnetic phase (which anticipates the most important features of the phase diagram). In Sec. IV, we analyze the phase diagram in terms of the relative strength of the aperiodic interactions. A summary of the main results and some conclusions are presented in Sec. V.

\section{FORMULATION OF THE PROBLEM}

We consider spin variables, $\sigma_{i}= \pm 1$, on the sites of a Cayley tree, with ferromagnetic $\left(J_{1}>0\right)$ interactions between first neighbors, and antiferromagnetic $\left(J_{2}<0\right)$ interactions between second neighbors along the branches of the tree (in Fig. 1, we draw some generations of a Cayley tree of coordination $z=3$ ). Now, we add extra second-neighbor in-

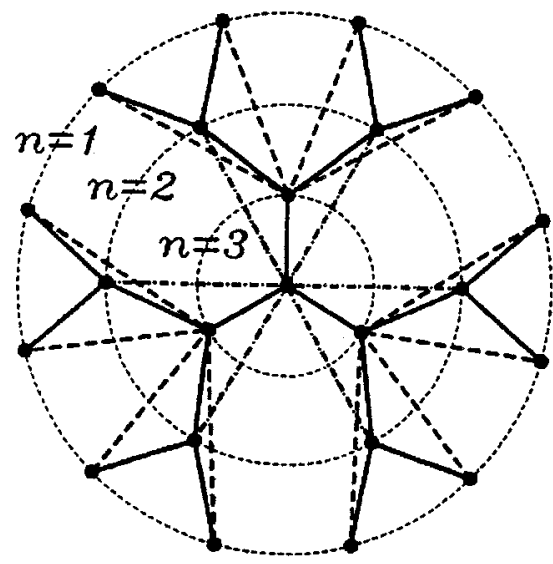

FIG. 1. Three shells of a Cayley tree with coordination $z=3$. The solid and dashed lines indicate the interactions between first $\left(J_{1}>0\right)$ and second neighbors $\left(J_{2}<0\right)$, respectively. The dotdashed lines indicate the sum of the periodic and the extra aperiodic interactions between second neighbors $\left(J_{2}+J_{F}\right)$. 


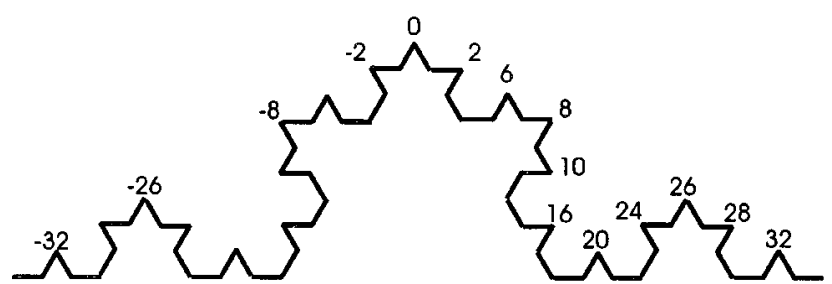

FIG. 2. Third step in the construction of the Koch curve. The sites located at the kinks of the fractal define an aperiodic sequence of integer numbers [they are selected by Eq. (1), and define an aperiodic sequence of second-neighbor interactions].

teractions $\left(J_{F}>0\right)$, according to an aperiodic rule. In this work, we use the Koch curve and the Fibonacci sequence to choose these extra aperiodic interactions.

In the nonbranching Koch fractal, the sites at acute angles define an aperiodic set along the chain (see Fig. 2). To select the pairs of second-neighbor interactions along the tree, we use the function

$$
t(i)=\delta(i, 0)+\sum_{s=1}^{\infty} \delta\left(\frac{4^{s}}{2}, i\left[\bmod 4^{s}\right]\right),
$$

which is +1 for sites corresponding to acute angles along the Koch curve, and vanishes otherwise [7].

The simple Fibonacci sequence of two elements, $A$ and $B$, is given by the inflation rule

$$
A \rightarrow A B ; \quad B \rightarrow A
$$

If we start with a single element $A$, the recursive use of these rules gives rise to a truly aperiodic sequence of $A$ and $B$ elements. To use this sequence to select sites with extra second-neighbor interactions, we define

$$
t(i)= \begin{cases}0 & \text { if } i \text { corresponds to an element } A \\ 1 & \text { if } i \text { corresponds to an element } B .\end{cases}
$$

Although keeping all the main features of the complex phase diagrams, the problem can be drastically simplified in the limit of infinite coordination of the tree $(z \rightarrow \infty$, while $z J_{1}$ and $z^{2} J_{2}$ remain finite). Now, as in the work of Yokoi et al. [3], we take advantage of this limit to write the map

$$
\begin{gathered}
X_{n+1}=\tanh \left[X_{n} / T+Y_{n}\right], \\
Y_{n+1}=X_{n}[r t(n)-p] / T,
\end{gathered}
$$

where $n=1,2,3, \ldots$ refers to a generation of the tree, $T$ is the temperature (in units of $\left.z J_{1}\right), p=-\left(z^{2} J_{2}\right) /\left(z J_{1}\right)$ is the parameter of competition, $r=\left(z^{2} J_{F}\right) /\left(z J_{1}\right)$ gauges the effect of the aperiodicity, and the function $t(n)$ is given by either Eq. (1), in the Koch case, or Eq. (3), for a Fibonacci rule. The essential point of this work consists in the introduction of $r$-mediated second-neighbor interactions whose aperiodicity is given by $t(n)$. The case $r \equiv 0$ corresponds to the original uniform model [3].

Along the lines of previous analysis of aperiodic systems [7], in this investigation we use a scheme of periodic approximations. According to this scheme, the aperiodic system is replaced by a set of periodic arrays, such that the length of the unit cell of each array increases with the order $G$ of the approximation. As $G$ increases, the aperiodic unit cell captures more and more features associated with the effects of aperiodicity. The behavior of the aperiodic systems is then obtained from the limit of the periodic approximations $(G \rightarrow \infty)$. To carry out the calculations, we replace $t(n)$ in Eq. (4) by $t_{G}(n)$, which restricts the aperiodicity in the model to the length $L_{G}$ of the unit cell in each periodic approximation.

In the case of the Koch rule, $G=0$ corresponds to a linear chain. $G=1,2,3, \ldots$ corresponds to successive stages in the construction of the fractal. The length of the unit cell in each stage is $L_{G}=4^{G}$. The function $t_{G}(i)$ is given by

$$
t_{G}(i)=\delta\left(0, i\left[\bmod 4^{G}\right]\right)+\sum_{s=1}^{G-1} \delta\left(\frac{4^{s}}{2}, i\left[\bmod 4^{s}\right]\right) .
$$

For the Fibonacci rule, the length of the successive unit cells is given by the Fibonacci numbers. Thus, the lengths $L_{G}$ are obtained from the iterations

$$
L_{G+1}=L_{G}+L_{G-1}, \quad \text { with } L_{1}=L_{0}=1 .
$$

According to Eq. (2), a linear chain of elements of type $A$ corresponds to $G=1$. The effects of aperiodicity first appear in the $G=2$ approximation. The function $t_{G}(i)$ is given by

$$
t_{G}(i)=\left\{\begin{array}{lll}
0 & \text { if } i\left[\bmod L_{G}\right] & \text { corresponds to an element } A \\
1 & \text { if } i\left[\bmod L_{G}\right] & \text { corresponds to an element } B .
\end{array}\right.
$$

\section{STABILITY OF THE PARAMAGNETIC PHASE}

To analyze the linear stability of the trivial attractor, $\left(X^{*}, Y^{*}\right)=(0,0)$, associated with the paramagnetic phase, we write the linear form

$$
\left(\begin{array}{l}
X_{n+1} \\
Y_{n+1}
\end{array}\right)=\left(\begin{array}{cc}
1 / T & 1 \\
(r t(n)-p) / T & 0
\end{array}\right)\left(\begin{array}{l}
X_{n} \\
Y_{n}
\end{array}\right) .
$$

Due to the existence of sites that interact in different ways with their neighbors, we have a site-dependent linear stability matrix, which assumes two different forms, $M_{0}$ or $M_{1}$, according to whether $t(n)=0$ or 1 . It is easy to see that

$$
M_{0}=\left(\begin{array}{cc}
1 / T & 1 \\
-p / T & 0
\end{array}\right)
$$

and

$$
M_{1}=\left(\begin{array}{cc}
1 / T & 1 \\
(r-p) / T & 0
\end{array}\right)
$$

with eigenvalues

$$
\lambda_{0}^{ \pm}=\frac{1}{2 T}[1 \pm \sqrt{1-4 T p}]
$$

and

$$
\lambda_{1}^{ \pm}=\frac{1}{2 T}[1 \pm \sqrt{1+4 T(r-p)}],
$$


respectively.

In the uniform case $(r=0)$, the stability region of the paramagnetic fixed point, coming from

$$
\operatorname{Abs}\left(\lambda_{0}^{+}, \lambda_{0}^{-}\right) \leqslant 1
$$

is given by

$$
T \geqslant 1-p \quad \text { if } p<1 / 2 ; \quad T \geqslant p \quad \text { if } p>1 / 2 .
$$

For $p<1 / 2$, the eigenvalues are real, and there is a transition between a paramagnetic and a ferromagnetic phase. For $p>1 / 2$, the eigenvalues are complex, and the transition is between a paramagnetic phase and a modulated structure with a continuously varying wave number.

In the aperiodic problem, the discussion of the stability depends on the eigenvalues $\lambda_{\infty}^{ \pm}$of a matrix $M_{\infty}$ that comes from the product of an infinite number of factors $M_{0}$ and $M_{1}$ in a well-defined (aperiodic) way. As the matrices $M_{0}$ and $M_{1}$ do not commute, the calculation of these eigenvalues is nontrivial, so we are forced to resort to numerical methods. Within the scheme of periodic approximations, we consider sequences of matrices, $M_{G}$, at all orders $G$, consisting of aperiodic finite strings of factors $M_{0}$ and $M_{1}$. The eigenvalues $\lambda_{G}^{ \pm}$of $M_{G}$ completely determine the stability region of the paramagnetic phase to order $G$ of the approximation.

There is a particular case that is amenable to some analytical calculations. For $r=p, M_{1}$ has one vanishing eigenvalue. In the basis where $M_{0}$ is diagonal (indicated by $\left.\bar{M}_{0}\right), M_{1}$ assumes the form

$$
\bar{M}_{1}=\left(\begin{array}{cc}
\lambda_{0}^{+}+p / S & \lambda_{0}^{-}-p / S \\
\lambda_{0}^{+}+p / S & \lambda_{0}^{-}-p / S
\end{array}\right)=\left(\begin{array}{cc}
\alpha & \beta \\
\alpha & \beta
\end{array}\right),
$$

where

$$
S=\sqrt{1-4 T p}
$$

From this particular form, we calculate the finite eigenvalue, and analyze the stability properties associated with all matrices $\bar{M}_{G}$. This case, which we plan to examine in full detail in a forthcoming paper, corresponds to the removal of a set of bonds from the original uniform model.

At each order of the periodic approximation, we have to evaluate the eigenvalues of the corresponding $2 \times 2$ matrix $M_{G}$. As the order $G$ increases, the matrix elements become high-degree polynomials in $p$ and $T$, and we are forced to resort to numerical calculations. However, the exact analysis of the lowest-order approximations already gives an overall picture of how the system behaves at higher orders. Let us consider the first two orders in the Fibonacci case. For $G=2$ and 3, we have $M_{2}=M_{0} M_{1}$, and $M_{3}=M_{0} M_{1} M_{0}$, respectively. The eigenvalues $\lambda_{2}^{ \pm}$of $M_{2}$ are given by

$$
\lambda_{2}^{ \pm}=\frac{1}{2 T^{2}}\left[1+r T-2 p T \pm \sqrt{(1+r T)^{2}-4 p T}\right] .
$$

These eigenvalues are real if $(1+r T)^{2} / 4 T \leqslant p$. As in the original uniform model, real and complex eigenvalues indi- cate the nature of the phase just below the paramagnetic border. Thus, provided that $r \leqslant 2$, the region of paramagnetic stability, given by

$$
T \geqslant \frac{r+\sqrt{r^{2}+4}}{2}-p \quad \text { if } p<\frac{r}{2}+\frac{r^{2}+2}{2 \sqrt{r^{2}+4}},
$$

and

$$
T \geqslant p \sqrt{1-r / p} \quad \text { if } p>\frac{r}{2}+\frac{r^{2}+2}{2 \sqrt{r^{2}+4}}
$$

undergoes minor changes with respect to the uniform case. However, if $r>2$, there are significant changes in the phase diagram. The curve given by Eq. (19) is not entirely contained in the region of complex eigenvalues. Thus, as $p$ increases above some threshold $p_{R}$, the line of transitions comes back to the region of real eigenvalues, and the paramagnetic phase is stable for

$$
T \geqslant p-\frac{r+\sqrt{r^{2}-4}}{2} \quad \text { if } p>p_{R}=\frac{r}{2}+\frac{r^{2}-2}{2 \sqrt{r^{2}-4}} .
$$

The appearance of real eigenvalues in the region that was formerly characterized by complex ones is the most important change introduced by the extra aperiodic interactions. In the first order of the approximation this new feature is restricted to large values of $r$, but at higher orders it is observed for all $r>0$. Indeed, the eigenvalues $\lambda_{3}^{ \pm}$of $M_{3}$ are given by

$$
\begin{aligned}
\lambda_{3}^{ \pm}= & \frac{1}{2 T^{3}}[1+r T-3 p T \\
& \left. \pm \sqrt{(1+r T-p T)\left(1+r T-5 p T+4 p^{2} T^{2}\right)}\right] .
\end{aligned}
$$

The existence of real or complex eigenvalues depends on the cubic polynomial in $T$ in the square root. The regions of real eigenvalues in the $p \times T$ plane are given by

$$
p \leqslant \frac{5-\sqrt{9-16 r T}}{8 T}
$$

and

$$
\frac{5+\sqrt{9-16 r T}}{8 T} \leqslant p \leqslant \frac{1+r T}{T} .
$$

The stability condition, $\left|\lambda_{3}^{ \pm}\right| \leqslant 1$, in the regions of complex eigenvalues is expressed as

$$
T \geqslant p[1-r / p]^{1 / 3} .
$$

In the region of real eigenvalues the stability condition depends on the roots of a cubic equation. Instead of presenting an analytical discussion of all cases, in Fig. 3 we draw the regions of real and complex eigenvalues as expressed by Eqs. (22) and (23), as well as the transition lines given by Eq. (24) for the particular value $r=0.1$. When the eigenvalues are real, the transition line was obtained by numerically evaluating the roots of a cubic equation. The new feature of 


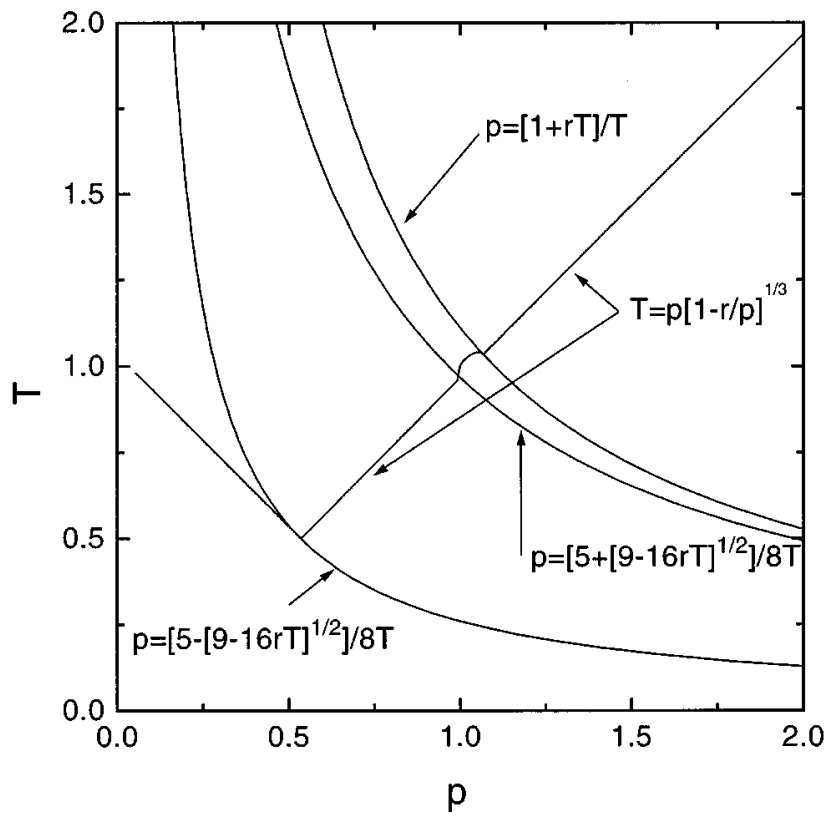

FIG. 3. Detail of the paramagnetic stability region of the phase diagram for the Fibonacci rule at the $G=3$ approximation. The regions of real eigenvalues, given by Eqs. (22) and (23), contain the border of the ferromagnetic phase and the bump associated with the $1 / 6$ phase.

this diagram is the presence of a small bump in the transition from the paramagnetic to a modulated phase [that is, in the region, given by Eq. (23), where the eigenvalues become real and Eq. (24) does not hold anymore]. The finite stripe with real eigenvalues is analogous to the infinite region with real eigenvalues in the former case $\left(p>p_{R}\right.$ for $\left.r>2\right)$. From Eqs. (22) and (23), it can be seen that the stripe with real eigenvalues increases with $r$. For $r \approx 0.8$, the bump is so large that it touches the descending branch of the paramagnetic line where the eigenvalues are real.

This kind of behavior is typical at all high-order approximations, although the details become increasingly difficult to work out exactly (due to the degree of the associated polynomials). For $G=2$ and $G=3$, we note that the changes in the real character of the eigenvalues depend on the second and third-degree polynomials under the square root in Eqs. (17) and (21). On the other hand, these expressions depend upon the traces and determinants of $M_{2}$ and $M_{3}$, which are given by twofold and threefold products of matrices $M_{0}$ and $M_{1}$. Due to the particular dependence of the elements of $M_{0}$ and $M_{1}$ upon $p$ and $T$, it is possible to show that the degree of the polynomial is given by the number of factors $M_{0}$ or $M_{1}$ in $M_{G}$ (hence, by the number of sites $L_{G}$ in the unit cell). If $L_{G}$ is odd, this polynomial may give rise to $\left(L_{G}-1\right) / 2$ stripes (as in the case of $M_{3}$ ). If $L_{G}$ is even, there can exist $\left(L_{G}-2\right) / 2$ stripes, besides an infinite region of real eigenvalues for large enough $p$ (as for $M_{2}$ ). For increasing values of $r$, there is a broadening of the stripes and the corresponding bumps. After a certain point, the bumps become so large that they start to push each other, and the aperiodic rule selects which of the bumps will either survive or be destroyed for large values of $r$. In the following section, we report a numerical integration of the map to analyze the mechanisms of phase selection.

\section{ANALYSIS OF THE PHASE DIAGRAMS}

We have performed numerical integrations of the map (4) to determine the dependence of the phase diagrams on the parameter $r$ and to investigate the convergence of the results obtained from the periodic approximations. We always calculate the largest Lyapunov exponent $\eta$ of the attractor, and identify the wave number $k$ (in units of $2 \pi$ ) associated with the dominant mode. The $p \times T$ plane has been scanned for $p \in[0.5,3.0]$ and $T \in[0.001,3.0]$, with a grid whose typical intervals are $\delta p \sim 0.001$ and $\delta T \sim 0.01$. A particular point $(p, T)$ is associated with a modulated phase if at least one neighboring point has a dominant mode with the same wave number (and a negative Lyapunov exponent). In the illustrations we draw only the most important phases.

In Fig. 4(a) we show a $p \times T$ phase diagram for the first Koch approximation, $G=1, L_{G}=4$, and $r=0.1$, which is similar to the case discussed in the previous section. The paramagnetic line displays only one single bump. All major phases of the uniform case $(r=0)$ are still present (and can be obtained according to a Farey summation rule [6]). The main difference with respect to the uniform case refers to the $k=1 / 8$ phase, which has become much broader, its upper part occupying the region of the bump. This phase-locking effect induced by the period of the larger unit cell is typical at all higher-order approximations. All bumps due to the presence of real eigenvalues, in a region otherwise characterized by complex eigenvalues, will be filled up by a single phase, at least for small values of $r$.

As the modulated phases keep their character up to the paramagnetic line, the selection rules for the wave numbers of the phases that fill up the different bumps should be determined from a more thorough investigation of the properties of the eigenvalues along the transition. Except for small values of $G$, it is not possible to carry out this analysis exactly. Then, let us use the $G=3$ Fibonacci approximation to illustrate the essential steps and arguments that can be extended to higher-order approximations.

First, we note that, in the uniform model, the imaginary part of the eigenvalue $\lambda_{0}^{+}$varies continuously from 0 to 1 as $p=T$ varies from $1 / 2$ to $\infty$. In this case, the effect of $M_{0}$ consists in rotating the vector $\left(\begin{array}{l}X_{n} \\ Y_{n}\end{array}\right)$, whose components are the local effective magnetizations per spin of two successive generations of the Cayley tree, by an angle $\theta=\sin ^{-1}\left[\operatorname{Im}\left(\lambda_{0}^{+}\right)\right] \in[0, \pi / 2)$. The wavelength of the modulated phase is given by $L_{0} V$, where $L_{0}$ is the distance between adjacent spins and $(V-1)$ is the number of nonequivalent sites between two successive equivalent sites. Thus, $V$ is also the number of factors $M_{0}$ that are required to bring $\left(\begin{array}{l}X_{n} \\ Y_{n}\end{array}\right)$ back to its original orientation, that is,

$$
V \theta=2 \pi
$$

In units of $2 \pi$, the dimensionless wave number is given by

$$
k=\frac{1}{V}=\frac{\theta}{2 \pi} .
$$

The eigenvalue $\lambda_{3}^{+}$, with $r=0$, describes the same situation as above. However, as $\theta \in[0,3 \pi / 2)$, we have 

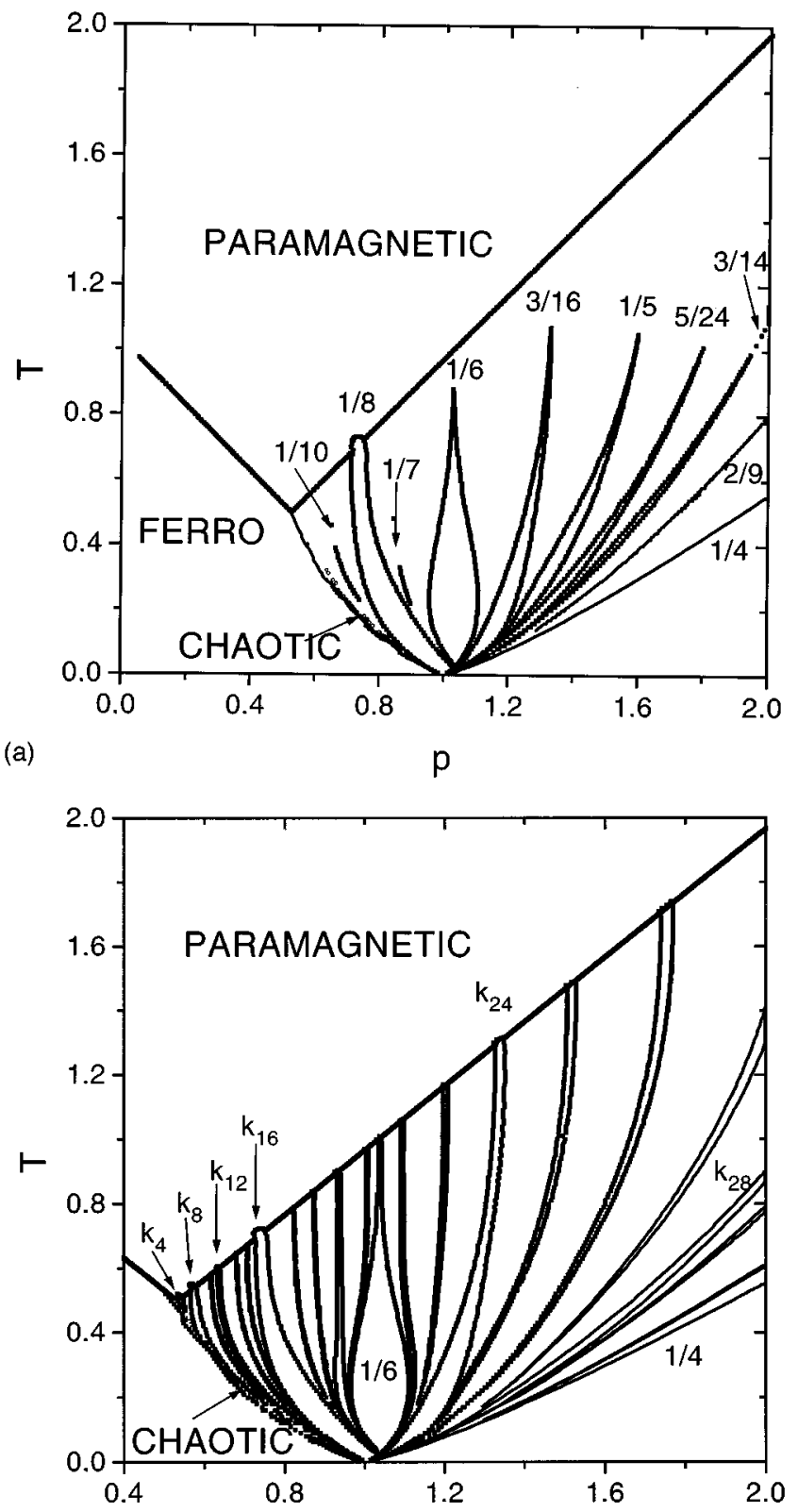

(b)

FIG. 4. Phase diagrams according to the Koch rule, for $r=0.1$, at different orders of the periodic approximation: (a) $G=1$; the only bump is occupied by the $1 / 8$ phase that lifts the $1 / 6$ phase from the $T=0$ axis. The main modulated phases close to the $1 / 8$ phase, as $1 / 10$ or $1 / 7$, become very thin (the superscript $G$ is omitted in the notation of the wave numbers); (b) $G=3$; the 16 bumps are more densely distributed in the region of small wave numbers. At the precision of the calculations, a unit cell with 64 aperiodic bonds already gives the main features of the $G \rightarrow \infty$ limit. We indicate a narrow region of chaotic attractors.

$$
k=\frac{\theta}{3 \times 2 \pi} .
$$

For $r \neq 0$, the situation is very much the same, except that phase locking occurs for $\theta=\pi$, that is, for real eigenvalues. The corresponding phase is associated with the wave number $k=1 / 6$, as can be checked numerically. A phase diagram as in Fig. 4(a) can be obtained by replacing the factor 3 in the

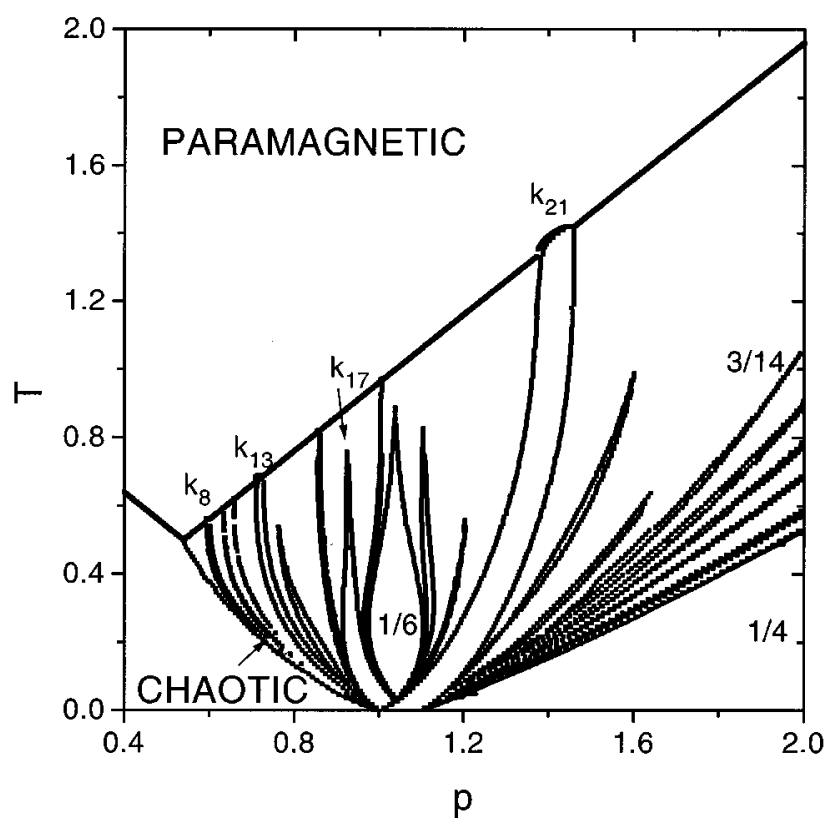

FIG. 5. Phase diagram according to the Fibonacci rule, for $r=0.1$, and $G=9$, corresponding to $L_{G}=55$. Notice the changes in the ground state $(T=0)$.

interval of definition of the angle $\theta$, and in the denominator of Eq. (27), by a factor 4, which is the length, in terms of $L_{0}$, of the unit cell at order $G=1$.

The analysis for larger values of $G$ is essentially the same. The angle $\theta$ belongs to the interval $\left[0, L_{G} \pi / 2\right)$, and the $\left(L_{G}-1\right) / 2$ bumps that appear in the stripes of real eigenvalues are filled up by phases with wave numbers given by

$$
k_{m}^{G}=\frac{m}{2 L_{G}}, \quad \text { with } \quad m=1,2, \ldots,\left(L_{G}-1\right) / 2
$$

The effect of higher-order approximations is illustrated in Fig. 4(b) for the Koch rule with the same value $r=0.1$, at order $G=3$, corresponding to $L_{G}=64$. A comparison with the diagram for $G=4, L_{G}=256$ (not shown here) indicates that, within the accuracy of the grid, we have already reached the $G \rightarrow \infty$ limit of a full Koch sequence. We note that, from the main commensurate phases of the original uniform model, only the $k=1 / 6$ phase survives. The other phases, especially in the region of small wave numbers bounded by the $k=1 / 6$ phase, have become much less relevant with respect to those with wave numbers $k_{m}^{G}$, given by Eq. (28), which occupy the bumps with real eigenvalues. As $L_{G}=4^{G}$, all phases from one given order of the approximation, associated with the wave numbers $k_{m}^{G}$, will also be present at all higher-order approximations.

This situation is similar for the Fibonacci rule. In Fig. 5 we show the diagram for the Fibonacci rule at order $G=9$, corresponding to $L_{G}=55$. Within the numerical accuracy of the calculations, these diagrams have already converged to the typical forms associated with the $G \rightarrow \infty$ limit. There are two major differences with respect to the Koch rule. First, the successive values of $L_{G}$ have no common factors, so that the phases $k_{m}^{G}$ change from one generation to the next. Nevertheless, we note that the changes in the wave number be- 


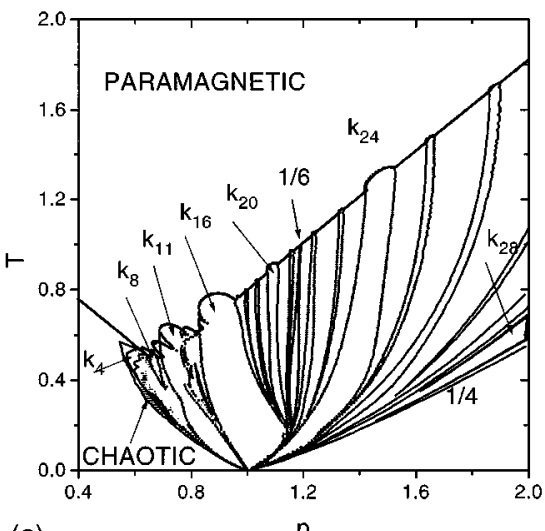

(a)

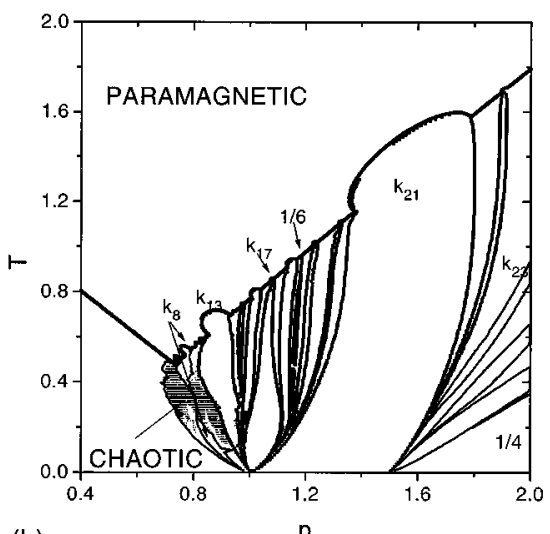

(b)

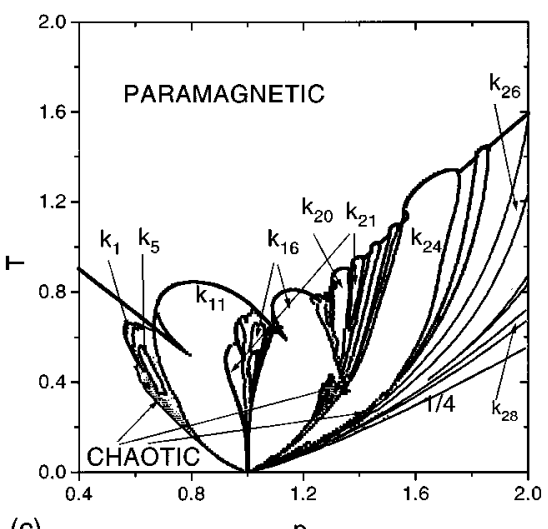

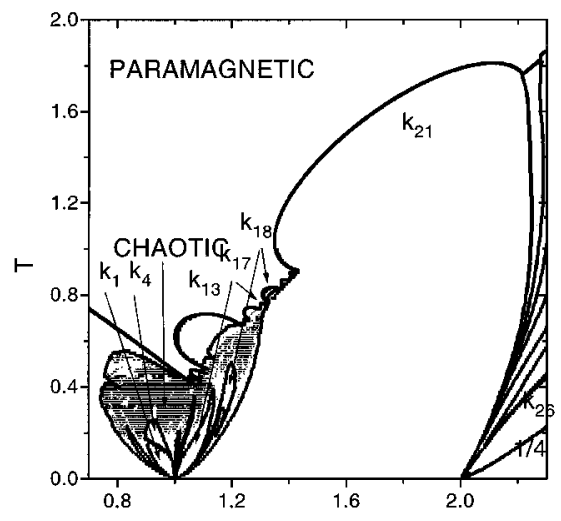

(d)

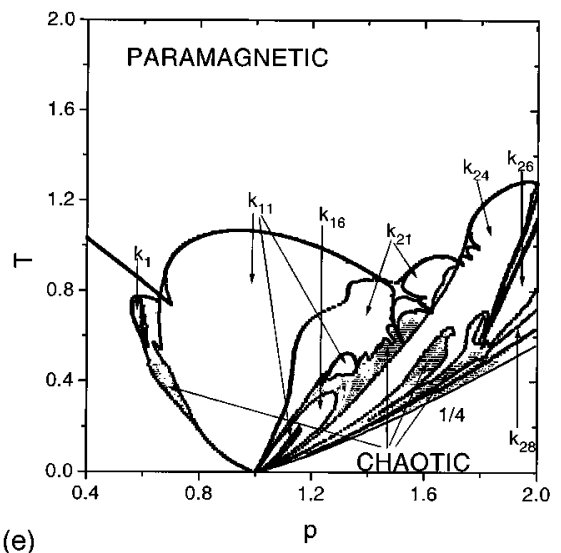

(e)

(c)

FIG. 6. Phase diagrams according to the Koch (at order $G=3$ ) and Fibonacci (at order $G=9$ ) rules, for increasing values of $r$ : (a) Koch rule for $r=0.5$; besides $k=1 / 4$, the dominant modulated phases are $k_{11}, k_{16}, k_{20}$, and $k_{24}$. The $1 / 6$ phase is greatly reduced; (b) Fibonacci rule for $r=0.5$; the $k_{21}$ phase dominates the diagram. Other relevant phases are $k_{13}$ and $k_{17}$. The chaotic region (indicated by small dots) has been considerably enlarged; (c) Koch rule for $r=1.0$; the dominant phases are still $k_{11}, k_{16}, k_{20}$, and $k_{24}$. There is a splitting of some regions (for example, $k_{21}$ appears in two distinct regions), and a new region of chaotic phases (for $p \sim 1.2-1.4$ ); (d) Fibonacci rule for $r=1.0$; the $k_{21}$ phase and the chaotic region dominate the diagram. There is a splitting of the other relevant phases $\left(k_{13}\right.$ and $\left.k_{17}\right)$; (e) Koch rule for $r=1.5$; the dominant phases are $k_{11}, k_{21}$, and $k_{24}$. Splitting and co-stability of phases are present in many regions of the phase diagram. The chaotic region extends up to $p \sim 1.6$.

come smaller for increasing $G$, and it is possible to follow this convergence towards well-defined limits. The second aspect refers to the existence of an interval of length $\Delta p=0.1$, along the $p$ axis, in the ground state $(T=0)$, between the ferromagnetic and the $1 / 4$ phases. The phase on this interval belongs to the set given by Eq. (28), as it extends upwards, at higher temperatures, until occupying a bump in the transition line. From the periodic approximations, we see that the associated wave number converges to $\phi^{2} / 2$, where $\phi=(\sqrt{5}-1) / 2$ is the inverse of the golden mean.

Now we analyze the dependence of the diagrams on the strength of the aperiodic couplings. In Figs. 6(a)-6(e), we show diagrams for different values of $r$, with $G=3$ and $G=9$, for Koch and Fibonacci rules. As should be anticipated, for increasing values of $r$ the system develops some new features, which are quite distinct from those of the original uniform model. For instance, the bumps become larger 
TABLE I. Limit values of some relevant structures of the phase diagrams according to the Koch and Fibonacci rules.

\begin{tabular}{lccccc}
\hline \hline Koch & $G=1$ & $G=2$ & $G=3$ & $G=4$ & $G \rightarrow \infty$ \\
\hline & $1 / 8$ & $3 / 32$ & $11 / 128$ & $43 / 512$ & $1 / 12$ \\
& $1 / 8$ & $5 / 32$ & $21 / 128$ & $85 / 512$ & $1 / 6$ \\
Fibonacci & $G=6$ & $G=7$ & $G=8$ & $G=9$ & $G \rightarrow \infty$ \\
\hline & $5 / 26$ & $16 / 84$ & $26 / 136$ & $21 / 110$ & $\phi^{2} / 2$ \\
& $4 / 26$ & $13 / 84$ & $21 / 136$ & $17 / 110$ & $\phi / 4$ \\
& $3 / 26$ & $10 / 84$ & $16 / 136$ & $13 / 110$ & $\phi^{3} / 2$ \\
\hline \hline
\end{tabular}

and the filling phases will start to compete among themselves. For $r=0.5$, as in Figs. 6(a) and 6(b), the phases $k_{m}^{G}$, with small values of $m$ such that $k_{m}<1 / 6$, occupy almost the entire region under the paramagnetic line. Some phases start to split into disconnected regions (for example, $k_{10}^{3}$ in the Koch case, and $k_{8}^{9}$ for the Fibonacci rule). Also, there appear regions of co-stability of attractors. Some modulated structures penetrate into the region of stability of the trivial paramagnetic fixed point, and even occupy the bumps associated with other phases. This is the case of the phase diagram for the Koch rule, with all trajectories starting from $(0.5,0.5)$. The diagrams for the Fibonacci rule were constructed with trajectories from $(0.1,0.1)$, so that the modulated phases do not penetrate into the region of (linear) stability of the paramagnetic fixed point. In both diagrams, the surviving 1/6 phase is now very slim. We also note that the regions of some $k_{m}^{G}$ phases become dominant over the neighboring phases (which is still more evident for larger values of $r$ ). It is important to follow the successive periodic approximations in order to extract the rule of formation of the relevant phases and to identify the remaining structures in the $G \rightarrow \infty$ limit. In Table I, we list some of the relevant phases in Figs. 6(a)-6(e) with their respective limit values. Figure 6(b) shows that the $\phi^{2} / 2$ phase occupies a segment of extension $\Delta p=0.5$ along the $T=0$ axis. Numerical tests confirm that this is the dominant structure of the phase diagram, with a segment of length $\Delta p=r$ at $T=0$. Finally, we observe a substantial increase in the region of chaotic attractors, which is even more pronounced in the Fibonacci case.

For $r=1.0$ [Figs. 6(c) and 6(d)], the aperiodic couplings cause further modifications in both Koch and Fibonacci rules. In general, however, the changes in the diagram of the Fibonacci case have been more dramatic. In both cases, several phases disappear, due to the enlargement of the regions of the dominant phases (see Table I). Other phases still survive, but they have lost their contacts with the paramagnetic transition line (that is, the corresponding bumps have disappeared). The number of important phases with large disconnected regions (for example, $k_{19}^{3}$ or $k_{21}^{3}$, in the Koch case, and $k_{17}^{9}$ or $k_{18}^{9}$, for the Fibonacci rule) is also a novel feature of the diagrams. In the Koch case, there is no intermediate phase along the $T=0$ axis between the ferromagnetic and the $1 / 4$ phase. In the Fibonacci case, the very large extension of the $\phi^{2} / 2$ phase produces a clear distinction between two regions. For $k<\phi^{2} / 2$, the aperiodic interaction dominates, with phases $k_{13}^{9}$, and $k_{17}^{9}$ or $k_{18}^{9}$, as well as a large chaotic region.
For $k>\phi^{2} / 2$, the phases are certainly distorted, although keeping some similarities with the uniform model. Also, the chaotic attractors tend to occupy larger regions of the phase diagram. In the Koch case, they appear for $k \sim 0.2$, whereas they were previously confined to the region $k \sim 0-0.1$. In the Fibonacci case, they occupy a very large extension, for $k<\phi^{2} / 2$, between the dominant phases (at low temperatures) and the paramagnetic transition line.

In the Fibonacci case the $r=1$ diagram already contains all main features that will also be present for larger values of $r$. In the Koch case, as shown in Fig. 6(e), we have to go to $r$ as large as 1.5 to see the dominant role of the aperiodic interactions. The characteristic feature is the domination of the $k_{11}^{3}$ and $k_{21}^{3}$ phases, which are proportional to the fraction of the number of sites with $r$-mediated interactions. From the diagrams, we see that the bumps corresponding to these phases tend to join each other, while the bumps associated with previously significant phases (for example, with the $1 / 8$ phase) have been destroyed. The extension of the regions of chaotic attractors has been substantially enlarged in comparison with the former situations.

\section{CONCLUSIONS}

We have investigated the effects of the inclusion of aperiodic interactions in the phase diagram of an analog of the ANNNI model on a Cayley tree. The problem is formulated as a two-dimensional nonlinear dissipative map whose attractors correspond to solutions deep in the interior of a large tree. We use a scheme of periodic approximations to find the behavior of the system under aperiodic rules generated by a Koch curve and a simple Fibonacci sequence. Some analytical and numerical calculations have been performed to obtain the region of stability of the paramagnetic phase and the main structures of the $T \times p$ sections of the phase diagram, for different values of the parameter $r$ of aperiodicity.

The appearance of some bumps in the modulated region of the paramagnetic transition line is one of the features of the phase diagrams that we believe to be new. We show that these bumps correspond to regions of real eigenvalues of the linear stability matrix of the paramagnetic phase. For small values of $r$, they are filled up by single phases, giving rise to a phase-locking phenomenon. We have obtained the rules for the number of bumps and the phase selection, in terms of the length of the unit cells of the periodic approximations.

For small values of $r$, the effects of aperiodicity are restricted to the regions of small wave numbers, corresponding to small values of the parameter $p$ of competition. For large $p$, the aperiodic interactions become less important and the diagram keeps the original structure. As the parameter $r$ of aperiodicity increases, there is a corresponding enlargement of the regions with relevant changes in the phase diagram. Also, there appear drastic modifications in the regions of small $p$ (or $k$ ), in which several phases are either wiped out of the diagram or split into disconnected regions. The emergence of large regions with chaotic orbits is a typical feature of aperiodicity for large values of $r$.

The analysis of models with two different aperiodic sequences, according to the Koch and Fibonacci rules, indicates some common features, such as the existence of bumps, the phenomenon of phase locking, and, for large $r$, 
the existence of a single structure that survives in the central part of the phase diagram, with a large chaotic region and some remnants of the old sequence of modulated phases. However, there are some differences. The most significant of them refers to the behavior along the $T=0$ axis. In the Koch case, the multiphase point of the original uniform model still survives at $p=1$, as the meeting point of the ferromagnetic $(k=0)$ and the $k=1 / 4$ phases. In the Fibonacci case, there appears a new phase along the $T=0$ axis, from $p=1$ to $p=1+r$, separating the ferromagnetic and the $k=1 / 4$ phases. This new structure, with wave number $\phi^{2} / 2$ in the limit of large aperiodic cells, extends up to the paramagnetic line and dominates the phase diagram for large values of $r$. This difference may be traced back to a special feature of the Koch case, as the length of the unit cell in any periodic approximation is a power of 4 . A rough look at the same model according to a generalized Fibonacci rule (generated by the substitutions $A \rightarrow A B^{n}$, and $B \rightarrow A$, with $n \geqslant 2$ ) indicates the presence of another phase between the ferromagnetic and the $1 / 4$ phases in the ground state.
[1] W. Selke, Phys. Rep. 170, 213 (1988); in Phase Transitions and Critical Phenomena, Vol. II, edited by C. Domb and J. Lebowitz (Academic Press, New York, 1992); J. M. Yeomans, in Solid State Physics, edited by H. Ehrenreich and D. Turnbull (Academic Press, San Diego, 1988), Vol. 41.

[2] J. Vannimenus, Z. Phys. B 43, 141 (1981); S. Inawashiro, C. J. Thompson, and G. Honda, J. Stat. Phys. 33, 419 (1983); A. M. Mariz, C. Tsallis, and E. L. Albuquerque, ibid. 40, 5777 (1985); C. R. da Silva and S. Coutinho, Phys. Rev. B 34, 7975 (1986).
[3] C. S. O. Yokoi, M. J. de Oliveira, and S. R. Salinas, Phys. Rev. Lett. 54, 163 (1985).

[4] H. Roeder and J. M. Yeomans, J. Phys. C 18, L163 (1985).

[5] S. Fishman and J. M. Yeomans, J. Phys. C 18, 857 (1985); P. Bak, S. Coppersmith, Y. Shapir, S. Fishman, and J. M. Yeomans, ibid. 18, 3911 (1985).

[6] M. N. Tamashiro, C. S. O. Yokoi, and S. R. Salinas (unpublished).

[7] R. F. S. Andrade and H. J. Schellnhuber, Europhys. Lett. 10, 73 (1989). 